\title{
Commercial Low-Altitude UAS Operations in Population Centers
}

\author{
Ella M. Atkins, ${ }^{1}$ Amar D. Khalsa, ${ }^{2}$ Mark D. Groden ${ }^{3}$ \\ University of Michigan, Ann Arbor
}

\begin{abstract}
Unmanned air systems (UAS) have numerous military, law enforcement, and commercial applications. Commercial potential in particular will not be realized until a comprehensive strategy is developed for safely and fully integrating UAS into the national airspace system over both rural and urban regions. This paper overviews a broad spectrum of UAS commercial applications to motivate airspace integration. Focus is then placed on use of small to micro-scale UAS in potentially lucrative but ultimately challenging benchmark applications: on-demand surveillance over a densely-populated urban region. Following the introduction of urban use cases, we present alternative criteria for small UAS safety that focus not on vehicle preservation but instead on avoiding collateral damage to people and property on the ground as well as to higher-cost airborne assets. We conclude the paper with a discussion of safety preservation when a micro air vehicle over an urban area encounters turbulence or wind shear, a likely day-to-day scenario that can introduce significant risk of a forced landing given realistic constraints on disturbance rejection. This study, led by Raytheon, is part of a NASA Research Announcement activity for Integrating Advanced Concepts and Vehicles into NextGen.
\end{abstract}

\section{Introduction}

$\mathrm{T}$ he pressing need to maximize commercial transport safety and capacity while minimizing environmental impact has resulted in a next-generation airspace system plan that focuses on two of three parts of the national airspace system: enroute airspace greater than FL100 and the airportal environment near runways. Large unmanned aerial systems (UAS) such as Global Hawk can fit within this structure as on-demand but infrequent operations. However, small UAS (SUAS) operations, where "small" is defined as a UAS under 35 pounds, will occupy airspace well under FL100 and would initially occupy uncontrolled airspace with no to extremely rare entry into congested airportal corridors. As part of the NASA Research Announcement (NRA) activities for Integrating Advanced Concepts and Vehicles into NextGen, Raytheon and its collaborators, including the University of Michigan, studied the impact of four future vehicle classes on NextGen in 2025 through 2040, including SUAS. This paper investigates the use cases and safety issues arising from SUAS operating in denselypopulated urban regions.

Currently small UAS operations are conducted with an FAA Certificate of Authorization (CoA) typically granted to entities seeking operations below altitudes GA craft would occupy. While appropriate for initial low-altitude UAS platform flight tests, the CoA does not provide the flexibility to operate commercially over evolving sites of public or individual interest. A limited number of operators, primarily in remote regions, have been granted permission to conduct UAS operations in designated test ranges. These remote ranges will provide valuable means to test operational protocols, but they will not support a class of on-demand UAS operations even approaching the freedom current GA and small commercial aircraft operators enjoy today. The primary cited impediment to UAS pervasively operating in the national airspace system (NAS) is increased risk, both real and perceived. Although early UAS maturation has occurred primarily in the DoD sector, if UAS-specific risk could be appropriately computed and controlled, regulatory, air traffic, and operational protocols can be introduced to flexibly accommodate rather than segregate UAS operations.

As occupants of primarily uncontrolled airspace, SUAS would share the skies with general aviation (GA) operations. So long as commercial and private UAS and GA activities remain sparse, it may be sufficient to define special-use areas for UAS, or to adopt a simple deconfliction approach such as defining a specific flight altitude for UAS that only must be verified clear when GA traffic transits during climb or descent. Emerging "sense-and-avoid" technologies will enable UAS to evade a nearby aircraft, and will ideally be sufficiently small and low-cost to be affixed in most UAS platforms. Given sufficient risk mitigation for UAS operations, we can begin to consider an alternative model, where business use cases predict rapid UAS proliferation in the private and commercial sectors to an equal or even greater extent than has been observed in military operations. In this scenario, either the FAA will

\footnotetext{
${ }^{1}$ Associate Professor, Aerospace Engineering Dept., University of Michigan, Ann Arbor, MI, Associate Fellow.

${ }^{2}$ Graduate Student Research Assistant, Aerospace Engineering Dept., Ann Arbor, MI, University of Michigan.

${ }^{3}$ Undergraduate Student Researcher, Aerospace Engineering Dept., Ann Arbor, MI, University of Michigan.
} 
maintain and extend regulations that stifle the potential for such UAS-centric businesses, or else new strategies must be developed and deployed with expedience to competitively support such operations.

This paper overviews potential small UAS (SUAS) applications that motivate proliferation of small UAS in lowaltitude airspace through a series of business use cases as well as associated flight plan characteristics. To provide context for the business use and safety discussions, we introduce a challenge problem. How do we operate small UAS at low altitudes in a densely-populated region such as Manhattan? Such operations have the potential to mix UAS with commercial and general aviation traffic, e.g., on approach to or departure from La Guardia, JFK, Newark, Teterboro or other nearby general aviation airport. Even if the UAS are segregated below commercial traffic, such operations will necessarily be conducted in close proximity to a significant number of people, requiring that the population not be exposed to unacceptable risk and that operations be conducted in a manner that does not increase levels of annoyance and distraction beyond those already present in the urban environment. We propose a concept of safety unique to the small, expendable UAS, focusing on minimizing risk to the environment rather than to the airframe itself. As a case study in maintaining safety in adverse conditions, we introduce a concept of operations for managing low-altitude turbulence and wind shear with a small helicopter UAS to highlight the distinction between vehicle preservation and our goal of "maintaining safety for people and valuable property".

\section{Small UAS Business and Physical Trajectories}

The surveillance UAS objective is to collect and disseminate information, not transport passengers or cargo. The popularity of websites such as Google Earth demonstrates our desire to view real Earth images rather than simply consulting a road map or aviation chart. The UAS can provide an unprecedented capability for applications that require real-time camera or sensor coverage of regions not within view of fixed cameras, or perhaps more uniquely the UAS can provide sensors that move in the environment. Such aerial mobility will allow the foreman to closely inspect a construction site, enable the scientist to follow real-time chemical or temperature gradients through the atmosphere, or more radically, endow a web-based spectator with the ability to travel via UAS data on a customized journey through a nearby or distant environment. Figure 1 provides a high-level summary of such operations, highlighting those that may be considered most "outside the box" with respect to established UAS applications but that also may have the greatest potential to proliferate both in number and diversity of operations managed through commercial enterprise. The "construction site inspection" scenario is categorized in the "single-user data collection" scenario, while science can require single or multi-user data perhaps in combination with "payload drop" to simultaneously characterize multiple areas of interest. The "entertainment" application area has not yet been developed commercially but has potential to connect with a large-scale audience by revolutionizing real-time webbased information sites.
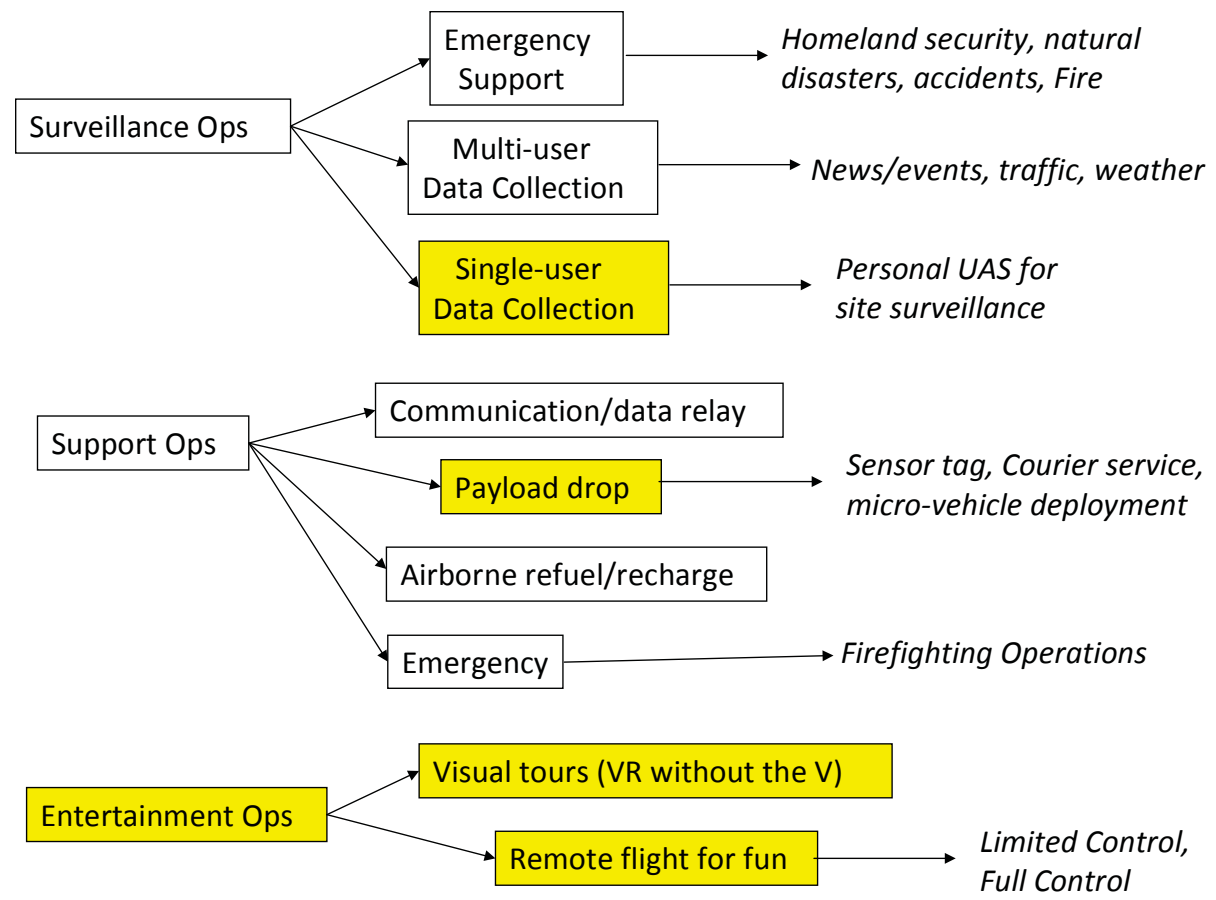

Figure 1: Existing and Potential UAS Applications (less traditional apps highlighted). 
Commercial small UAS operations would not use existing runways thus would not occupy arrival or departure slots. They would, however, require support infrastructure, such as launch and retrieval sites, recharge stations, and maintenance and storage facilities. For an urban setting, establishing base stations on high-rise rooftops would minimize the need for additional infrastructure. Surveillance operations would also require use of communication frequency bands, requiring appropriate allocation and support from the FCC. Customer hiring and dispatch of the UAS may be fully-automated, but each launching UAS will be entering the airspace thus will need to maintain separation from other manned and unmanned traffic.

Urban UAS operations can require a variety of sizes and scales, but all share common baseline requirements: the need for administrative and command \& control facilities, ability to manage ultra-large-scale datasets produced by the UAS, archived for future reference, and made available to customers. Launch/retrieval sites may be fixed or mobile with easy access to maintenance, storage, and recharge stations. While these 'ground support' needs are formidable, they can be met with existing technology so long as a business case is sufficiently promising. Below is a business case summary to motivate commercial SUAS urban operations. We believe urban operations have potential to obtain the highest profit margins given the level of dynamicism and number of citizens who would value real-time data of their neighborhood (with data post-processing prior to distribution to prevent violations of privacy).

\section{A. Small UAS operations in Manhattan}

Consider an operational scenario targeting the Manhattan borough of New York City, one of the most denselypopulated regions of the US. Airspace segregation is a viable option for Manhattan given geography and existing airspace restrictions. Low-altitude general aviation and small business aircraft operate over a Hudson River corridor, except for isolated heliport facilities in the city. Commercial aircraft operate out of three major and several small-midsize airports around Manhattan, but such traffic would overfly Manhattan only after climbing well above high-rise structures. This protocol creates a "bubble" around the Manhattan land mass (with "notches' for heliport rotorcraft entry/exit). Small UAS could operate in this bubble without risk of collision with manned or high-value unmanned aircraft. Risk analysis, then, can focus primarily on the potential for collateral damage to people and valuable property on the ground and methods to minimize risk of such damage. Under this operational paradigm, Manhattan represents a "simple case" where airspace segregation may in fact be possible without major changes to existing manned operations. On the other hand, Manhattan operations directly challenge our notions of real/perceived risk managed today by "open area overflight" protocols. Privacy and public acceptance challenges are also critical to appropriately overcome.

In Manhattan or more generally any urban region that can support UAS operations, a combination of single-use and regular customers would comprise the UAS user base. Given the necessity to operate within acceptable risk constraints, initial operations would likely be conducted by approved commercial urban UAS operators rather than "any private citizen or public entity with means to buy and fly a UAS". Ideally, early operations would guide regulatory agencies toward practical protocols based on actual urban operational experiences as well as simulation analyses. Certified commercial operators would be motivated by profit, and will likely reap maximum profit by catering to a variety of users. Single-use customers might request photo or video data at a specific date/time, whether a vacationer in a bright shirt waves from the Empire State Building or a local resident requests an overhead view of their favorite place in Central Park in good weather during peak fall color. Courier service (local document delivery) could be organized as a commercial entity with regular and single-use customers. Large-scale commercial customers such as the news media would likely arrange certain regularly-scheduled operations (e.g., traffic monitoring) augmented by single-use operations (e.g., to gather overhead footage of a special event in Central Park). This class of customers would need data ranging from high-quality live video to weather and atmospheric composition sensors for meteorologists. Emergency service operators would not be regularly-scheduled but would require rapid response in large numbers to support situational awareness and communication relay needs associated with an emergency event.

Although one could consider single-use and media operations at equal or lower priority than private manned aircraft operations, critical emergency service UAS operations could arguably rise to an equal or even greater priority than commercial transport operations. In the segregated Manhattan UAS airspace "bubble", such priority tradeoffs may not be necessary; generally such tradeoffs will require careful regulatory consideration.

\section{B. Urban mission scenarios}

Consider a single-use mission where a UAS is deployed to a series of popular Manhattan restaurants, perhaps to check the wait lines extending into the street with a low-altitude fly-by. If the restaurants are all in the same neighborhood, one UAS could handle the operation with a flight plan automatically-generated by a traveling salesman problem (TSP) algorithm derivative. Flight trajectories can adopt one of two basic strategies to fly 
between sites, as shown in Figure 2: a climb above building tops followed by a straight-line route to the urban canyon in which the next site is located, or a path through the perpendicular network of streets of "Manhattan distance" length. The choice will depend on the distance the UAS is from the target site and the location of the restaurant within the street grid. For example if the target site is one block north and 10 blocks east, it would be more efficient to fly the one block north and then fly the straight 10 blocks within the urban canyon, while if the route has substantial length along N/S and E/W directions, the climb to a direct route would be advantageous.

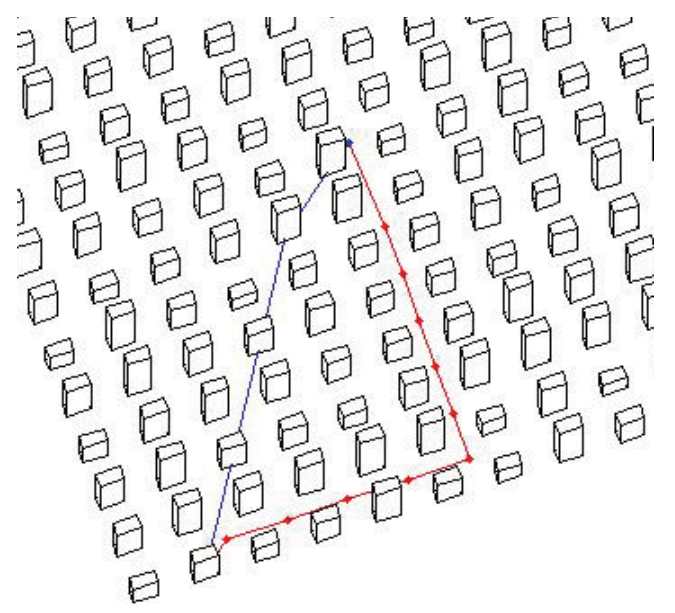

Figure 2: MATLAB Manhattan vs. Straight-line distance Simulation.

Different operations, even for a "restaurant observation scenario", will require different flight plan characteristics. UAS can fly-by to obtain a snapshot of waiting customers or loiter to observe how quickly the line moves or changes in length over time. For the fly-by, an efficient UAS operation would have multiple targets of interest and plan an efficient path between them. For loitering operations the vehicle should point to its surveillance target as continuously as possible and maintain a safe but proximal station relative to the target site. For additional operations such as the courier flight or the virtual tour, a waypoint-based approach is likely the more efficient method, although with courier service constraints on payload may require ordering the waypoints in "pickup-drop" pairs. Examples of alternative commercial scenarios of small UAS are be found below, in the context of a singleuser ("Jerry") who wants to stay informed through a "UAS information provider". The "restaurant surveillance" scenario is presented first followed by other examples that motivate both the business case and shed light on the types of risk that may be encountered during flight operations.

\section{Scene 1-Personal UAS Operation: Check the lines at popular lunch restaurants}

Jerry is sitting in his office at 11:30 on a Friday, planning a lunch with a number of co-workers as is their weekly tradition. He is having an energizing day and decides to plan lunch at one of the new popular restaurants that he's recently read about in the New York Times. The typical wait at these restaurants, however, is 45 minutes and none take reservations for lunch. Being popular, the phone lines at these restaurants are always busy, and web sites are far from updated. Since Jerry and his colleagues only have a short break for lunch, he logs onto rentaUAS.com to receive real-time location information and chooses this new restaurant as well as alternates.

To do this Jerry enters the site and visits the 'live view' section. Two of his location selections have already been requested by renters and are immediately available for "live view". Both of these locations appear to have waits significantly longer than is acceptable. Two SUAS are dispatched to the remaining un-monitored locations, one of which had been launched from the nearest base location and one which had been completing another "live view" request. The first UAS has only a two minute ETA. This information is now available for his viewing after he had observed the first two feeds. Although the restaurant's website indicates otherwise, by observation he finds that this restaurant has been temporarily closed.

The second UAS is launched from the base location atop a nearby rooftop. This UAS is approximately two miles from the selected location and has ETA of five minutes at $30 \mathrm{kts}$. As this second UAS arrives at the location it appears to be Jerry's lucky day, he sees on "live view" the restaurant is open and there is no significant line, thus Jerry has found his lunch venue. Given the group's limited lunch time constraint, Jerry requests that the UAS loiter 
or periodically revisit the site until his group arrives. He and his colleagues monitor UAS-supplied updates on their iPhones during the cab ride to lunch in case they need to divert to an alternate due to a last-minute "lunch rush".

\section{Scene 2 - Emergency UAS Operation: Provide early views of an accident with possible injuries}

As Jerry is getting out of his cab for lunch, he hears tires squealing and looks up just in time to see a car rear-end an SUV. Jerry, being a good citizen, decides that a call to the NYPD would be appropriate. Upon receiving this call the NYPD immediately dispatches their closest unit, but because it is lunchtime and the streets are crawling with people and gridlocked cabs, the nearest unit is 10-15 minutes away. Therefore the NYPD decided to deploy a UAS to evaluate the scene and decide which emergency vehicles are most appropriate. They request elicitation of a small VTOL UAS, which is launched from the roof top base of operations. Their central location enables reaching any site in Manhattan in five minutes or less. Unlike the commercial UAS feeds with obscured faces, license plates, etc., the NYPD UAS provides full-resolution video through a secure channel should specifics on vehicles and persons involved be needed later. From the video feed, the NYPD are able to discern that one of the vehicles is disabled and will need to be towed, and that they will need at least two ambulances for the car's vehicle's driver and passenger. As a result of the use of Emergency UAS Operations, the NYPD are able to dispatch the necessary emergency vehicles more rapidly and collect important site data before officers could even arrive.

\section{Scene 3 - Integrated Airspace Operations: Acquire detailed real-time coverage of commuter traffic}

Jerry has had a long week at the office and is ready to go home to New Jersey for the weekend along with the thousands of other commuters exiting the city. He decides to check real time traffic information on his favorite route out of the city. This request is handled by rentaUAS.com using a number of large fixed wing UAS's, but to provide detailed information requires overflight outside the Manhattan streets themselves. Jerry initially is pleased to see that over 20,000 other New York commuters had requested live UAS surveillance of bridge and highway traffic on his route home - this meant very low cost to be "just another data subscriber". However, Jerry is then informed some delay will occur due to a conflict with a business jet out of Teterboro carrying 4 passengers.

Is it appropriate to delay the UAS and leave thousands of people without real-time traffic information when they most need it? Or should the business jet be delayed an additional five minutes instead so that the UAS can be launched immediately, providing 20,000+ commuters with data to aid in their travel efficiency? Current policy would not allow a UAS in the area since the airspace is in close proximity to local busy airports. A next-generation strategy might allow the UAS to enter the airspace, provided risk concerns are adequately addressed and an open slot exists. However, existing metrics that consider capacity and runway queue efficiency do not apply to UAS. Economic, efficiency, and other metrics that account for information acquisition as well as passenger/cargo transport will be required to address equity and allocation questions in a manner translatable to operational practices.

\section{Scene 4 - Entertainment UAS Operation-Night visual tour of Manhattan}

When Jerry arrives home, he furiously prepares for the arrival of his sister's family. His nephew is fascinated by New York City and Jerry wants him to experience as much of the city as possible. Jerry, however, is not excited about driving back into the city for a second time in one day. Instead, Jerry purchases a night-time visual tour of Manhattan and surrounding points of interest through the camera of a UAS. He now looks on rentaUAS.com and sees that there are a number of 1 hour prescheduled tours that he can pick from, so he decides on the $9 \mathrm{pm}$ tour which will give his nephew enough time to arrive and get comfortable.

This tour is provided by mid-sized VTOL UAS with a maximum endurance of one hour and a maximum speed of $21 \mathrm{kts}$. This UAS is chosen so as to provide flexibility in the speed of the tour and allow the tour to provide hover views of the city highlights such as the Statue of Liberty, for which it files an explicit flight plan to avoid conflict with GA operations. Jerry selects this tour and chooses to watch it on a large TV screen to provide the best entertainment for the entire family. After the live tour is scheduled, the UAS is cleared for an appropriate flight route, and is then launched from a heliport located on the Hudson River. This UAS then provides a live night visual tour around the city and gives views once only accessible by in manned helicopter tours. Jerry's nephew records the video to take home, indicating this was the best souvenir ever.

\section{Scene 5 - Public Event UAS Operation-A concert in Central Park}

Jerry's family elects on staying in town - two of their favorite artists are playing back-to-back in a live Central Park concert. Although the whole family plans to attend the event, they also want as much coverage of the experience as possible for future enjoyment. To Jerry's surprise, rentaUAS.com denies his request to collect an overhead video of the concert. A major entertainment company has purchased exclusive coverage rights and will offer their own high-quality aerial as well as ground-based video footage available for customers at a modest price. 
A premiere UAS operator is hired by the entertainment company to gather comprehensive footage. This operator develops a mission plan in which multiple craft cover the event from multiple vantage points, traversing and zooming in on points of interest, and flying in shifts for the expected three-hour event. Jerry's nephew manages to attract the attention of a UAS camera during his favorite song, becoming a part of the video as well as experiencing the concert itself. A single emergency services UAS also loiters over the concert. Unobtrusive during routine monitoring, this UAS enables a central security office to monitor the crowd for potentially dangerous situations. This concert unfolds with no injuries or arrests. If an issue had arisen, the security UAS would have been ready - to detect, assess, and assist with information gathering throughout the incident.

The above scenarios, while not possible today, can be realized provided we establish appropriate procedures and a level of trust in UAS established through analysis and a record of safe operations. The remainder of this paper focuses on SUAS risk and safety issues to overcome, and discusses a challenging turbulence/wind shear situation in which the SUAS must execute a forced landing in a densely-populated urban area.

\section{Practical Safety Issues: Protect the People and Property, not the UAS}

Low-altitude small UAS surveillance in urban areas poses unique risks to safety. Unlike manned aircraft and high-value UAS, the small UAS may be considered expendable. This shifts safety concerns from protecting the vehicle itself to instead ensuring the SUAS brings no harm to valuable assets (or people) in its vicinity. The primary two risks associated with small UAS operations are: (1) Separation assurance / collision avoidance with larger manned or transport platforms and (2) Collateral damage to objects or people on the ground. Figure 3 illustrates a causal diagram that illustrates how vehicle, operational, and separation failures feed our primary SUAS categories, with distinction between injury and loss of life in the Figure.

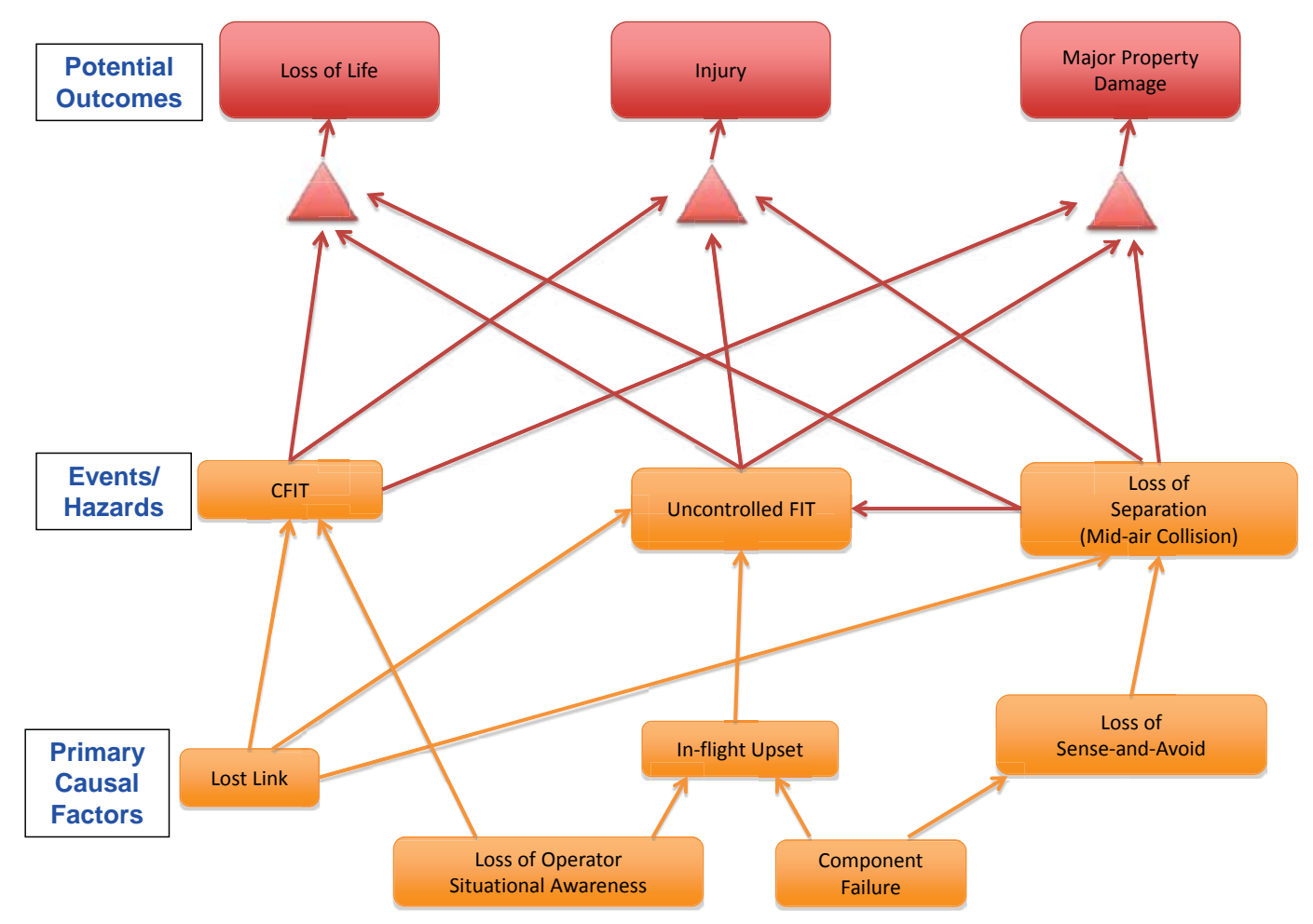

Figure 3: SUAS Risk Mapping

At appreciable altitudes certain airspace regions will be congested by transport operators on approach or departure to/from a proximate runway. At lower altitudes not along an approach or departure corridor, the UAS must deconflict with each other and GA aircraft. This "third region" of airspace (controlled or not) must be designed to accommodate SUAS operations - this airspace class can push the frontiers of automated separation assurance and 
deconfliction rather than force-fitting legacy GA policies on a new generation of flight platforms. While risks associated with losing separation are important, this paper acknowledges such risks but instead will focus on the risks of operating limited-reliability small UAS in close proximity to people and valuable property on the ground.

If all UAS systems are operating properly, ground-based assets are safe so long as the UAS doesn't "fly into terrain". With a large number of UAS operations, it is inevitable that the occasional UAS will experience a hardware or software failure while over a populous region. In fact, since the vehicles will likely be low-cost and light-weight to maintain economic competitiveness, UAS are highly unlikely to have anywhere close to the same reliability as commercial transports. UAS must therefore have multiple safe means of ditching when failures occur, even in an urban setting. These ditching strategies must be applicable to all adverse scenarios that are sufficiently likely and that introduce sufficient risk if experienced. Increasing the reliability of UAS to a degree in which ditching does not need to be considered would be extremely costly. It would also decrease the efficiency of the UAS due to the additional payload of multiple back up airframe, sensor, actuator, and computer systems. If designed to be robust, safe ditching strategies are more practical and cost effective. We note that the small-to-micro UAS conducting surveillance activities are of a scale more similar to a child's toy than even the smallest Cessna, as illustrated in Figure 4, by a GWS Dragonfly helicopter with weight of 15 ounces, a main rotor diameter of 20 inches, and a cruise speed of approximately 25 miles per hour. To minimize weight and cost, such vehicles are primarily constructed from "frangible" materials such as plastic with less potential to inflict damage should impact occur.

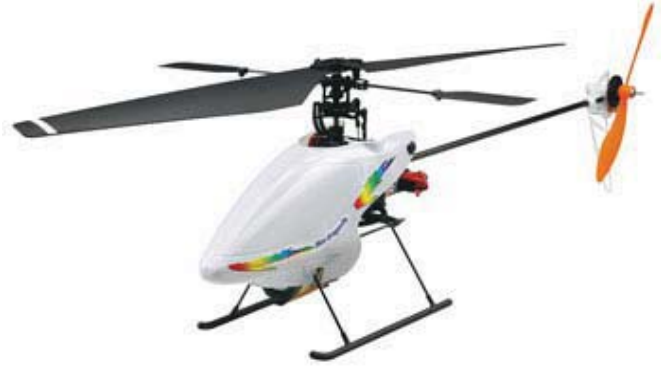

Figure 4: GWS Dragonfly

Urban operations with an energy-efficient, quiet, frangible UAS platform have the potential to be unobtrusive, particularly in a noisy environment such as Manhattan. If a frangible vehicle collides with any structure of reasonable strength, the platform disintegrates while the structure remains undamaged. Safety, then, can still be guaranteed so long as any UAS impact is with robust structures. In an urban environment, robust structures would include buildings, paved or unpaved ground, trees, and even cars/trucks (although a UAS impact into a car roof might be slightly startling). Below, we examine the concept of safety through three scenarios in which a frangible UAS in an urban environment guides itself to a "safe ditching" rather than guaranteeing no impact will occur.

Continuing our Manhattan theme, consider a micro-helicopter UAS surveying an area around Central Park that loses all thrusting power at an AGL altitude of 300 feet. The UAS immediately disengages the drive motor to enter autorotation, and the UAS sensors (or onboard data) indicate there are trees within autorotation range. The UAS proceeds to crash into the top of a tree in Central Park. Although requiring retrieval as would a child's wayward kite the UAS successfully avoids exposing bystanders to unacceptable risk. In another scenario, consider a UAS flying through city streets between buildings to survey the restaurant crowd, when left and right cyclic control is lost. The UAS recognizes its inability to continue its flight and begins searching for a safe means to ditch. A brick building is located along the default path, and the UAS increases forward speed to maximize its impact velocity with the building. Upon impact, the UAS is broken into many small fragments that fall to the sidewalk below. This UAS ditching mode has a goal of ensuring a collision of sufficient momentum occurs for the vehicle to decompose into harmless fragments. As a final scenario, consider a Statue of Liberty survey during which the UAS loses its tail rotor. Although onboard video is garbled due to rapid angular motion, the UAS determines based on its map that it may be over a large crowd of people and cannot land safely. With limited directional control, the UAS opts to climb at its maximum possible rate to allow the wind to push it over the water. Once safely over the water the UAS cuts all thrust and ditches into the water. In this last scenario, clearance from GA aircraft may be required, although a frangible MAV may impose negligible risk to even a modest GA craft.

Below, we delve into a models and data of use to SUAS ditching. Then, we explore in more depth a case where an SUAS emergency ditching is required due to adverse winds rather than SUAS electromechanical failure. Though ditching provides a risk mitigation solution in the scenarios presented in this paper, it is possible for the UAS to encounter a situation it does not recognize. So long as some flight control is possible, the UAS can ditch itself, but 
if total control is lost, the UAS may in fact descend without authority. As long as this descent is extremely rare, occurs in a vehicle the size of a child's toy, and the populous accepts the occasional frangible UAS "falling from the sky", even this worst-case situation will introduce annoyance but not grave risk. We are confident these goals are attainable, and that the next-generation urban population will notice a low-flying "toy" no more than a passing taxi.

\section{A. Models and Data for Emergency Management}

Aviation safety planning is of key importance to the safe operation of manned aircraft, providing human pilots with the proper tools to keep the manned vehicle safe, or minimizing risk to people and property regardless of UAS safety. Projecting SUAS behavior requires knowledge of UAS performance and its operating environment. So long as the UAS is operating normally, focus can be placed on efficient flight between job sites and high-quality data acquisition once onsite. When a situation occurs that may require urban ditching, worst case for a UAS that must not damage people or property, the SUAS must rapidly consider its alternatives in the context of itself and its environment, and select an appropriate path to a safe ditching site.

To define a suitable emergency route information must be quickly assimilated, particularly given rapid response time requirement and limited SUAS ground operator support. Although direct operator intervention may not be feasible, a datalink to the vehicle can provide environmental information such as temperature and humidity (for icing), observed convective activity, and cloud cover in the local operating area. This data can in turn be used to assess current aircraft performance, properly diagnose the disturbance condition in the context of the environment, and react accordingly. This information can also be used to determine what may be encountered along each potential flight direction and altitude, ranging from wind predictions to appreciable icing or convective activity. We presume a local map of man-made and natural terrain has been stored onboard, included uncertain models describing expected locations of people and valuable property.

From the human pilot perspective, available data is used to make an initial ditching location decision based on selecting a minimum-risk direction and angle of flight. To illustrate this concept, we define a situation awareness (SA) cylinder, abstracted into regions classified by expected environmental risks and potential for collateral damage on the ground when ditching in the corresponding flight direction. Figure 5 illustrates the simple SA cylinder concept. In an urban canyon environment, following a particular flight path and heading vector may result in safe or unsafe impact with a building or street, vectoring away from population (e.g., into a remote area of Central Park), or following a flight path likely to escape the adverse local condition (e.g., strong crosswind when traversing an open perpendicular street/corridor). The SA cylinder has no fixed diameter or height but instead represents projections of expected or worst-case conditions should flight be conducted along a particular [straight] path. In cases where nearby buildings or known hazardous conditions preclude flight operations, certain flight paths may be eliminated from consideration, while the rest are ranked primarily based on safety considerations, secondarily based on [expendable] UAS survival, and finally based on continued mission accomplishment (in cases where in-flight recovery is highly probable).

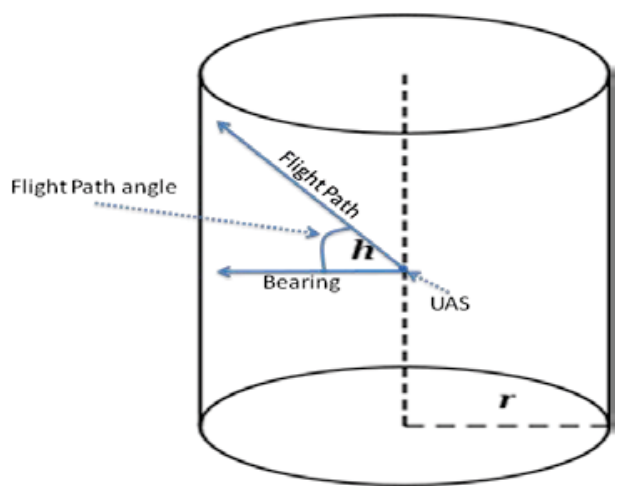

Figure 5: Situational Awareness (SA) Cylinder

The SA cylinder risk minimization approach described above can be partially mapped in the context of general aviation. In both cases, the pilot quickly determines which heading to fly, and how to climb/descend. GA pilots also use information such as expected weather, terrain characteristics, and vehicle capabilities. One notable difference, when comparing the UAS with "older" GA equipage, is that the GA aircraft will likely have little to no datalink capability and may not have an onboard computer capable of projecting expected performance. The GA pilot therefore must rely on their immediate observations and knowledge of historical trends, with respect to 
performance, atmospheric conditions, and terrain. The GA SA cylinder will therefore have a more limited quantitative extent with additional qualitative extent based on the pilot's experience and instinct. The primary stakeholder for GA flight, the pilot, is now solely responsible for the aircraft with some assistance provided by air traffic control. This puts a much higher importance on the pilot's ability to adapt to the environment, as opposed to the UAS flying lower but compiling more information into emergency handling decisions. Note, however, that while even SUAS are expected to possess high-rate datalinks, onboard processing capability for expendable platforms may be highly limited. This requires limited data processing and projection for real-time decisionmaking. Autonomous decision-making tools such as that required to direct a UAS to a safe ditching, while in principle may not require complex logic, still must be robust, except for extremely frangible platforms that do not impose risk despite the location or speed at which they impact terrain.

\section{Diagnosis and Handling of an Emergency: Wind Shear/Turbulence in an Urban Canyon}

When an SUAS finds itself in a severe wind condition that could force an unplanned landing, it is important to minimize risk to people and valuable property. ${ }^{1}$ To properly diagnose a turbulence or wind shear situation and the correct recovery procedure, we consider a model inspired by human pilot procedures that can be translated into a straightforward decision algorithm for SUAS. At the base level, the pilot attempts to translate adverse conditions to an appropriate reaction strategy and quantifiable values critical to anticipate vehicle performance thus guide recovery. ${ }^{2}$ The majority of current wind shear research has been directed towards the takeoff and landing phases of aircraft flight due to the extreme danger these operations pose due to their low altitude. Though not directly applicable, diagnosis and recovery methods designed for commercial and GA operations can inspire an analogous low altitude SUAS protocol. Wind shear or severe turbulence can be diagnosed by excessive change in climb rate $\dot{h}$, roll rate $\dot{\varphi}$, pitch rate $\dot{\theta}$, yaw rate $\dot{\psi} \cdot{ }^{3}$ Recovery procedures from turbulence or wind shear vary depending on vehicle type and performance envelope. For the purpose of this discussion, we focus on recovery procedures for small UAS helicopters. This SUAS would transition from a normal restricted flight envelope to an unrestricted maximum performance flight envelope to compensate as well as possible to the wind shear/turbulence event. ${ }^{4}$ Normal flight operations typically command trim states and transitions between trim states, where a trim state holds airspeed $V$,

pitch angle $\theta$, roll angle $\varphi$, and climb rate $\dot{h}$. Transition to the unrestricted flight envelope enables aggressive 3D flight maneuvering during which acquired payload data may be of poor quality but that maximizes likelihood of recovery and escape from the wind shear/turbulence condition.

Due to the need to keep development costs low and onboard navigation-related sensing to a minimum, SUAS may not have well-understood flight dynamics. Instead of the traditional multi-year design, analysis, and flight test cycles required to certify a new manned airframe, SUAS performance might instead be rapidly estimated at lowercost through existing models extrapolated to the vehicle's small scale. ${ }^{5}$ Thus, while a flight envelope can be approximated, actual performance would be expected to vary, with associated uncertainties potentially increasing loss-of-control risk. In the context of the inertial SA cylinder, flight path projections given current flight envelope constraints and observed winds may restrict the reachable headings and flight path angles substantially relative to nominal values. Drawing analogy to piloted aircraft protocol, approximate envelope estimates can provide parameter constraints such as "never exceed" values for airspeed, pitch angle/rate, and roll angle/rate. During a turbulence/wind shear event, guidance mode will shift from following the mission/flight plan to recovering attitude stability (even with aggressive control commands) and maximizing climb rate (to minimize/stabilize descent).

A tradeoff between exceeding structural airframe limits and arresting undesirable motions exists. Although marginal violation of envelope constraints may be acceptable, exceeding never exceed values by a large margin can result in an unrecoverable flight state or an in-flight breakup. An autonomous SUAS, therefore, must be capable of deciding how to balance aero/structural overload with attitude instability to effectively respond to a severe turbulence event. For example, if a turbulent condition induced an excessive descent rate $h_{e x-}$ or an excessive airspeed at an altitude $h_{A G L}>1000 \mathrm{ft}$, it would be most important to reduce airspeed to avoid structural damage and then arrest $\dot{h}_{-}$. This decision would be based on altitude (thus risk of flight into terrain) as well as structural limits. If this same condition occurred at an $h_{A G L}<100 \mathrm{ft}$, descent $h_{-}$must be first arrested regardless of structural loads to avoid flight into terrain; if the arrested descent is successful airspeed $V$ can then be cautiously reduced (provided it wasn't opportunistically reduced during control of descent). ${ }^{6}$ The relative priorities of meeting critical state value constraints therefore change dynamically based on risk minimization. Although in this example we focus on a procedure to avoid flight into terrain, an alternative strategy might purposely direct the UAS into an expedient descent, provided a safe ditching site has higher probability of being accurately targeted than the UAS has in recovering a stable flight condition. 
The choice of whether to attempt a turbulence/wind shear escape procedure versus completing an emergency landing relies on a number of factors including expected UAS performance limits, current vehicle state, and the severity and locality (size) of the turbulence or wind shear condition. Below we discuss a simple decision algorithm based on best (maximum) achievable climb rate, as well as procedure to execute a safe ditching should this rate indicate such action is required.

\section{A. Decision-making as a function of shallowest descent rate $h_{\max } \cdot$}

Maximum climb rate $h_{\max }$ (or shallowest descent rate) is a particularly important quantity since altitude is necessarily lost over time, and SUAS conducting urban operations typically will operate at low altitudes to provide high-quality information. A recovery procedure to achieve maximum climb (shallowest descent) varies depending on performance, ability to re-establish a stable, advantageous flight attitude, and the magnitude of a vertical downward wind component. Additionally, the decision to maximize climb rate may be secondary to avoiding overstress conditions, as discussed above. Consider an example where $\dot{h_{-}}, \dot{\varphi}$, and $\dot{\theta}$ all have undesirable (large magnitude) values. At high AGL altitude, the UAS would initially correct $\dot{\varphi}$ then $\dot{\theta}$ before power is applied to achieve $\dot{h_{+}}$. While in a $h_{A G L}<100 \mathrm{ft}$ turbulence situation, the UAS would either elect a safe ditching site (and continue its descent to the ground with priority on minimizing impact energy), or if overflying valuable or unknown assets the UAS would switch to unrestricted flight mode to maximize climb performance available regardless of the potential for structural damage.

\section{B. Real-time Reaction Constraints}

Most turbulence or wind shear situations will have little effect on flight safety, although they may degrade data quality or require alteration to the UAS flight plan. These situations must be distinguished from occurrences that require immediate action to avoid flight into terrain. To accomplish this the SA cylinder described previously can be decomposed into regions, each of which has an associated reaction time (urgency) rating. Relevant values including altitude $h_{A G L}$, distance to a nearby valuable object $d_{o b j}$, pitch and roll rates $(\dot{\theta}, \dot{\varphi})$, and aircraft inertial velocity with components $V_{\text {lateral }}$ and $\dot{h}$. If $h_{A G L}<100 \mathrm{ft}$ or $d_{o b j}<100 \mathrm{ft}$ and difficult turbulence/wind shear was predicted to continue, immediate action would be required. If $h_{A G L}$ and $d_{o b j}<500 \mathrm{ft}$ (a moderate distance) and turbulence/wind shear urgent but not immediate action would be required, enabling the craft to briefly deliberate its options. If $h_{A G L}>1000 \mathrm{ft}$ and $d_{o b j}>1000 \mathrm{ft}$ the UAS could remain in normal flight mode and compensate if conditions worsen. If any of $\dot{\theta}, \dot{\varphi}, V_{\text {lateral }}$, or $\dot{h}$ exceed maximum limits, urgent or immediate action is required, depending on the level to which limits are exceeded and the continued rate of change (desirable vs. adverse). Monitoring and contingency response planning actions might be invoked is if $\dot{\theta}, \dot{\varphi}, V_{\text {lateral }}$, or $\dot{h}$ parameters were in the vicinity but not substantially outside normal operating limits. As an example of reaction time use, consider a UAS flying straight down an urban canyon with obstacles on either side and below. If a wind disturbance induces appreciable roll and/or lateral motion, or if wind shear induces appreciable downdraft, immediate or urgent reaction would be required, depending on projected time to impact and ability of the vehicle to actively correct its flight path.

\section{Emergency Approach and Landing}

The primarily goal of a small UAS emergency landing is to minimize damage to people or valuable property that may be in the landing area. A secondary goal, especially for small but not frangible UAS, might be to minimize damage to the vehicle on landing so it can later be recovered and recycled/repaired. This emergency landing approach presumes the UAS is expendable thus safety is maintained so long as impact is only with objects of greater strength and/or minimal value. For the simplest and lightest UAS $(<1 \mathrm{lbs})$ emergency landing operations will be aimed at directing the vehicle's kinetic energy vector toward a safe impact zone, prioritizing the likelihood of landing in a safe zone over kinetic energy magnitude minimization. If the UAS is equipped with dangerous actuators, such as exposed propeller/rotor blades these should be locked or folded (depending on design) prior to ditching to minimize chance of blade strike or separation damage. Frangible SUAS can generally ditch without additional equipment. For applications in which frangible construction is not possible, additional systems such as a parachute might be required to bring kinetic energy to within safe constraints.

The decision of which direction to fly for ditching would primarily be based on risk analysis given an updated situational awareness (SA) cylinder. Other factors, such as ability to change flight direction before impact, or confidence of risk values in each potential ditching direction, can also be of use. When flying through an urban canyon, ditching the vehicle into a building given uncertain knowledge of people/property on the street below can be the lowest-risk solution. With sufficient altitude, the UAS could instead direct its energy to a remote area of Central 
Park, or to a ditching site in the East River. Although this discussion focuses on selection of ditching site and simple path to that site, it is also important to note that with sufficient altitude, localized urban canyon conditions might be averted by directing the UAS toward a site where turbulence conditions are significantly less severe. Should this site be heavily-populated, errors in predicted turbulence/wind shear predictions may be problematic. Application of an uncertain reasoning system that minimizes risk over expected wind and ground asset state as well as aircraft performance characteristics will likely provide the most comprehensive and informed outcome.

\section{Conclusion}

This paper has studied the business opportunities and safety challenges associated with operating SUAS in an urban environment. Commercial, government, and ultimately private operators will only be able to deploy UAS once both real and perceived risks have been addressed. We have presented a series of urban use cases for SUAS, and provided an alternative description of safe operations in the context of collateral damage. This approach allows loss of expendable SUAS without loss of safety, enabling low-cost, lightweight SUAS platforms to focus on new operational procedures to mitigate risk rather than redundant equipment. Full accommodation of UAS operations is not yet an integral part of the proposed Next-Gen airspace system, although the missions they could accomplish are relevant to a large cross-section of the population. We will define and solve problems associated with UAS/SUAS operations only when we embrace the potential for small UAS to be supported in our air transportation and surveillance system.

\section{Acknowledgements}

This research was supported in part under a subcontract from Raytheon Corporation, principle investigator for NASA NRA contract NNA08BA47C. The authors would like to thank team collaborators for the insightful discussions related to SUAS safety and operations, particularly Dan Salvano and James Luxhoj.

\section{References}

${ }^{1}$ Lissaman, P., "Effects of Turbulence on Bank Upsets of Small Flight Vehicles," AIAA Aerospace Sciences Meeting Including The New Horizons Forum and Aerospace Exposition, Baltimore, 2009.

2 Chang, H.P., Camp, D.S., Frost, W., McCarthy, J., "Wind Shear Characterization," AIAA $24^{\text {th }}$ Aerospace Sciences Meeting, Nevada, 1986.

3 Greene, R.A., "The Airborne Detection of Low Level Wind Shear," AIAA Aircraft Systems and Technology Conference, Los Angeles, 1978.

${ }^{4}$ Yavrucuk, I., Kannan, S., Prasad, J., Schrage, D., "Simulation Evaluation of a Reconfigurable Flight Controller of a Heli-UAV for Extreme Maneuvers," AIAA Modeling and Simulation Technologies Conference, Denver, 2000.

5 Stanzione, K., Randall, S., "Development of Generic Helicopter Performance Equations an Methodology," AIAA Aircraft Design, Systems and Operations Meeting, Baltimore, 1991.

${ }^{6}$ Stratton, D.A., Stengel, R.F., "Probabilistic Reasoning for Intelligent Wind Shear Avoidance," AIAA Guidance, Navigation, and Control Conference, Portland, 1991. 